\title{
The analysis of the electric vehicle charging infrastructure in Tyumen city
}

\author{
Anastasia Gorbunova ${ }^{1,{ }^{*}}$ and Ilya Anisimov ${ }^{1}$ \\ ${ }^{1}$ Tyumen Industrial University, 38, Volodarskogo str., 625000, Tyumen, Russia
}

\begin{abstract}
The development of electric vehicles, which is also observed in the Russian Federation at present, leads to the need to create a charging infrastructure. Variable operating conditions, and in particular low ambient temperature, cause difficulties in the use of electric vehicles, which are associated with a low power reserve and increased energy consumption in the winter. These features of the electric vehicle operation in the Russian Federation can lead to an increase in the number of charging stations needed to create a developed infrastructure, and, therefore, can increase capital costs. The purpose of this research is to identify patterns of the electric vehicle charging station operation in the regional infrastructure using the example of Tyumen city to develop a methodology for calculating their quantity that can satisfy demand in variable climatic conditions with a low capital cost. As a result of this study, patterns of change in the number of charging sessions, its duration and the amount of energy transferred to electric vehicles from the ambient temperature were obtained.
\end{abstract}

\section{Introduction}

Road transport is one of the main sources of air pollution in cities, which leads to the need to develop more environmentally friendly types of vehicles or to change approaches to moving people [1 - 2]. Currently, electric vehicles are the rapidly developing type of environmentally friendly vehicles in the world. As of July 1, 2019, their number in the world exceeded 5.1 million units.

In the Russian Federation, in order to reduce emissions of harmful substances into the atmosphere from road transport, along with the transition to electric vehicles, the development of vehicles with gas-balloon equipment is being considered. However, the transition to electric vehicles in the Russian Federation is already being observed, and this is a global trend, which necessitates the development of charging infrastructure. These trends are presented in the automotive industry development strategy of the Russian Federation, which also indicates that the creation of a developed network of charging stations is a prerequisite that contributes to an increase in the number of electric vehicles.

According to the data of the analytical agency Deloitte, more than $60 \%$ of potential buyers of electric vehicles identify the presence of a developed charging infrastructure as an important factor. In accordance with international experience in the development of

\footnotetext{
*Corresponding author: burakova.1992@mail.ru
} 
charging infrastructure, it was found that in the Netherlands, the commissioning of 2500 stations in the period from 2011 to 2012 led to an increase in the number of electric vehicles in operation. The introduction of an infrastructure development program for electric vehicles in Norway between 2010 and 2012 also caused an increase in the share of electric vehicle sales.

Currently, the most developed charging infrastructure is located in the Netherlands, where the number of charging stations per $100 \mathrm{~km}$ of roads is 19.3 units. In the Russian Federation, this indicator is 0.1 charging stations per $100 \mathrm{~km}$ of roads. Moreover, the largest number of charging stations are located in the big cities of the Russian Federation. This is in Moscow and in St. Petersburg. In the regions, this process is only in its infancy. The largest number of charging stations are located in Tyumen and Vladivostok, but this value does not exceed 10 units.

When the considered infrastructure is created, the limited number of charging stations is a feature of the stage of the emergence of the electric vehicle fleet. However, the low level of the electric vehicle adaptability for operation in low-temperature conditions, which are characteristic of the Russian Federation, leads to a decrease in their power reserve, which causes an increase in the number of charging stations and an increase in capital costs. Thus, the purpose of this research is to study the features of the existing regional charging infrastructure in the Russian Federation using the example of Tyumen city to identify patterns that will contribute to the creation of a methodology for calculating the number of charging stations when the considered infrastructure is in its infancy under variable climatic conditions.

The second section of this article discusses existing scientific approaches to the location and calculation of the number of objects serving vehicles, as well as presents methods for studying the source data. The third section shows the research results. In conclusion, the prospects for the development of this work are considered [1-18].

\section{Methodology}

The formation of scientific approaches to calculating the number and location of the charging infrastructure of electric vehicles, in particular buses and cars, has been observed over the past 15 years. Moreover, in the Russian Federation, these approaches have not yet been developed for electric vehicles. The calculation of the number of infrastructure facilities serving vehicles is considered in the works of A. A. Velnikovsky, S. V. Garkushina, I. M. Koklina, D. G. Asadov, I. F. Malenkina, and others [3 - 5]. In these studies, automobile gas filling stations are mainly studied as objects, taking into account factors such as regional characteristics of the territories, technological, technical and organizational parameters of the process of using NGV fuel in transport.

In the paper of A. A. Velnikovsky, the forecast of the development of vehicles with gas equipment is considered and a scientifically based approach to calculating the number and location of gas filling stations with a perspective up to 2023 is developed, taking into account the fleet of vehicles with gas equipment in use, average consumption of gas vehicles by trucks and buses, the time required for refueling and capital costs for creating a NGV station network. The developed methods have been introduced into gas engine fuel development programs in the regions of the Russian Federation.

A. A. Evtifeev was also involved in the development of a methodology for constructing a network of automobile gas-filling stations. He created the methodology of calculation and location of considered facilities based on an algorithm for solving a mathematical model of a gas refueling system for vehicles with compressed natural gas, taking into account factors affecting the determination of the expediency and form of organization of gas engine fuel supply various settlements. 
The calculation of the number of infrastructure facilities for servicing vehicles was carried out in paper of D. G. Asadov, where the minimization of capital and operating costs was considered as the objective function when creating the infrastructure for the technical service of mobile power units used in agriculture. At the same time, as a subsystem of the technical service, the author studied the charging infrastructure and performed its optimization taking into account the number of mobile power units, the operating modes of charging posts and the features of the operation of freight vehicles (mass of transported cargo at low speeds). The approach proposed by the author is based on the theory of queuing systems, in which he considers the consistency between the flow of applications and the intensity of service as the objective function.

Also studies aimed at developing methods for the rational location of objects serving vehicles, in particular electric vehicles, were identified in the analysis of previously paper. Distinctive features of electric vehicles during their operation are a reduced range in comparison with traditional vehicles and a long charging session. Therefore, the location of charging stations takes into account the pedestrian accessibility of the centers of gravity, the minimum distances between charging stations, which reduces the time spent on their search [6-15].

Minimization of operating costs is considered as the objective function in the research of Ko J., Shim J. S. The model for the location of taxi battery exchange stations presented in this paper takes into account not only the maximum power reserve, service time, but also the demand at each location, capital costs and the distance to the nearest points of attraction of the population where taxis can be called. Measurement and inclusion in the model of the distances between the taxi demand points and the estimated locations of the battery exchange stations is a prerequisite for calculating this model, since the distance and time of the vehicle moves between these centers involves additional operating costs and reduces the efficiency of the transport process. However, the application of this model is difficult for the location of slow charging stations, which require pedestrian accessibility of attraction centers. In this case, the authors of many researches proposed using models of maximum coverage of demand, in particular, it is presented in the study by Gimenez - Gaydou D., Ribeiro A., Cutierrez J., Antunes A. The objective function of this model is to maximize the coverage of emerging demand. As a prerequisite for the calculation, the coverage distance is used, which, as a rule, should be equal to the maximum walking distance to the nearest center of attraction. The input to the model is the demand arising at various nodes of the urban street-road network. Moreover, the coverage distance and demand are interconnected in accordance with the following rule: if the demand in the node is at a distance from the estimated location of the infrastructure object, which is less than the coverage distance, then demand is considered covered, otherwise not. As a result of this model, an arrangement of infrastructure objects is found that corresponds to the maximum value of demand covered by them. This approach, as a rule, is used to calculate only the optimal locations of slow charging stations, since it does not take into account the operating costs incurred by electric vehicle owners when searching for a free charging station, and the capital costs of installing it.

High capital costs can arise when installing fast charging stations in the city. In this case, scientists consider rational options for their location, taking into account the minimization of the cost of their creation. This model is presented in the paper of CruzZambrano M., Corchero C., where the input data are the matrix of population correspondence and the location of roads and existing infrastructure facilities serving vehicles. As a result of this study, the authors conclude that it is necessary to locate charging stations mainly on the territory of existing gas filling complexes in the city, taking into account the minimum intensity of the application flow. 
An important feature of these methodologies presented in foreign sources is the previously known number of charging stations that need to be distributed in the city. It is not aimed at calculating their number, taking into account the characteristics of the operation of electric vehicles.

Thus, the purpose of this article is to study the features of the existing regional charging infrastructure in the Russian Federation using the example of the city of Tyumen to identify patterns that will contribute to the creation of a methodology for calculating the number of charging stations when the considered infrastructure is in its infancy under variable climatic conditions.

\subsection{Research methodology}

In this research methods of regression and correlation analysis were applied to identify patterns. The source data was obtained using the PlugShare service and PJSC "SUENKO", which owns charging stations located in the city of Tyumen. The obtained dependencies will later be used to calculate the number of charging stations located in the cities of the Russian Federation.

\section{Discussion of results}

Currently, the city of Tyumen has the most developed regional network of charging stations. It includes 7 objects of charging infrastructure, of which 3 are "fast" and 4 are "slow" charging stations. The stages of commissioning charging stations in the city of Tyumen are presented in figure 1.

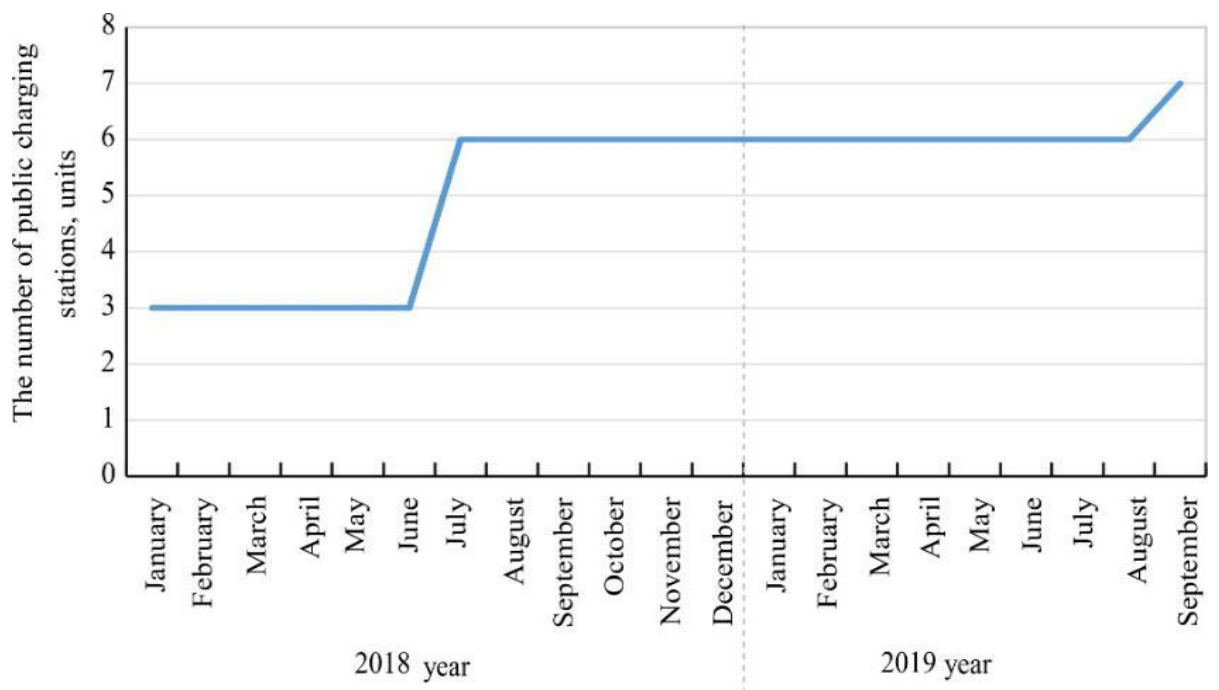

Fig. 1. Stages of commissioning charging stations in the city of Tyumen.

The increase in the number of charging stations contributed to the growth of the fleet of electric vehicles by more than 31 times. The dynamics of the number of electric vehicles charged from public charging stations in the city of Tyumen is presented in figure 2. 


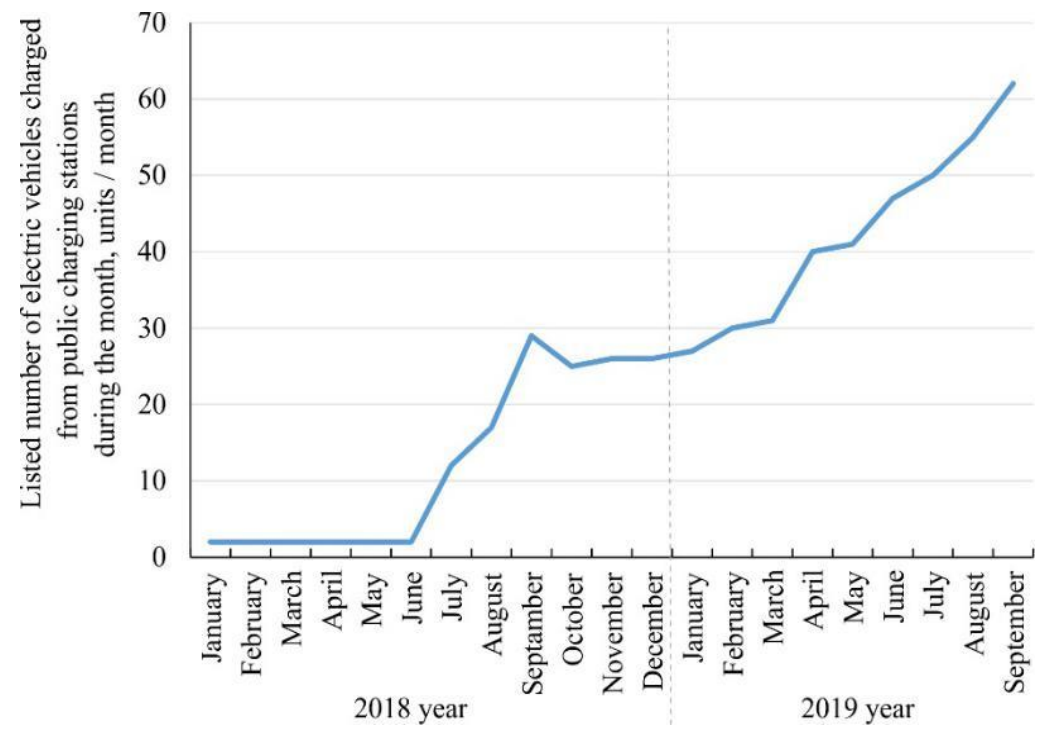

Fig. 2. The dynamics of the change in the number of electric vehicles charged from public charging stations in Tyumen.

An increase in the fleet of electric vehicles contributed to an increase in the number of charging sessions performed by stations during the month, which is shown in figure 3 . However, during the analysis of the data presented, it was revealed that in winter period there is a decrease in the number of running sessions by $40-47 \%$. At the same time, the number of electric vehicles operated in the winter period does not decrease, which is confirmed by the data presented in figure 2 . Therefore, to identify the reasons for the decrease in the number of charging sessions during the winter months, we analyzed data characterizing the parameters of the stations, namely the duration of the operation and the amount of energy consumed during its implementation.

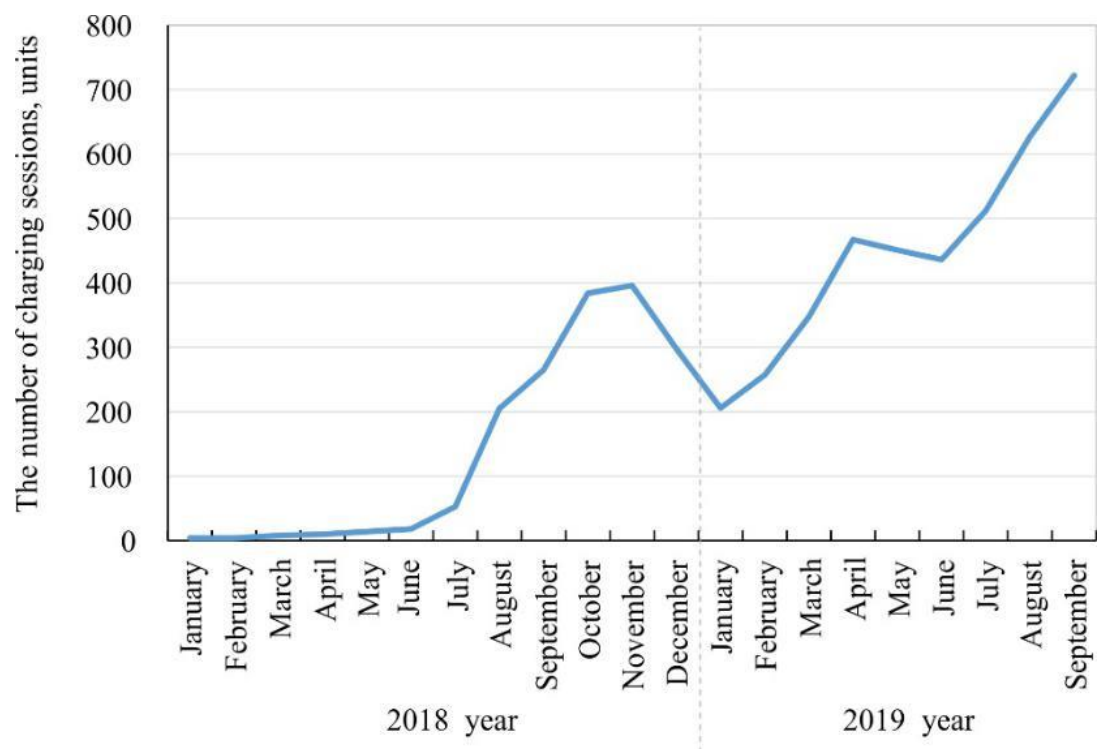

Fig. 3. The dynamics of changes in the number of charging sessions at public charging stations in the city of Tyumen. 
Additionally, the indicator of the number of charging sessions of one electric vehicle during the month, which is shown in figure 4, was calculated to confirm the absence of the effect of a decreasing number of electric vehicles in the winter period on the number of charging sessions.

With an increase and stagnation (from September 1, 2018 to February 1, 2019) of the number of electric vehicles charged from charging stations, as shown in figure 2 , a decrease in the number of charging sessions leads to the formation of a "failure" in the winter period of the charging session number that are performed by one electric vehicle for a month, which is shown in figure 4.

In the course of this study, the pattern was also developed for the change in the number of charging sessions from the ambient temperature, which is shown in figure 5. Processing of the initial data allowed us to establish a close connection between the identified input and output parameters. The pair correlation coefficient was 0.92 with a significance level of 0.95 .

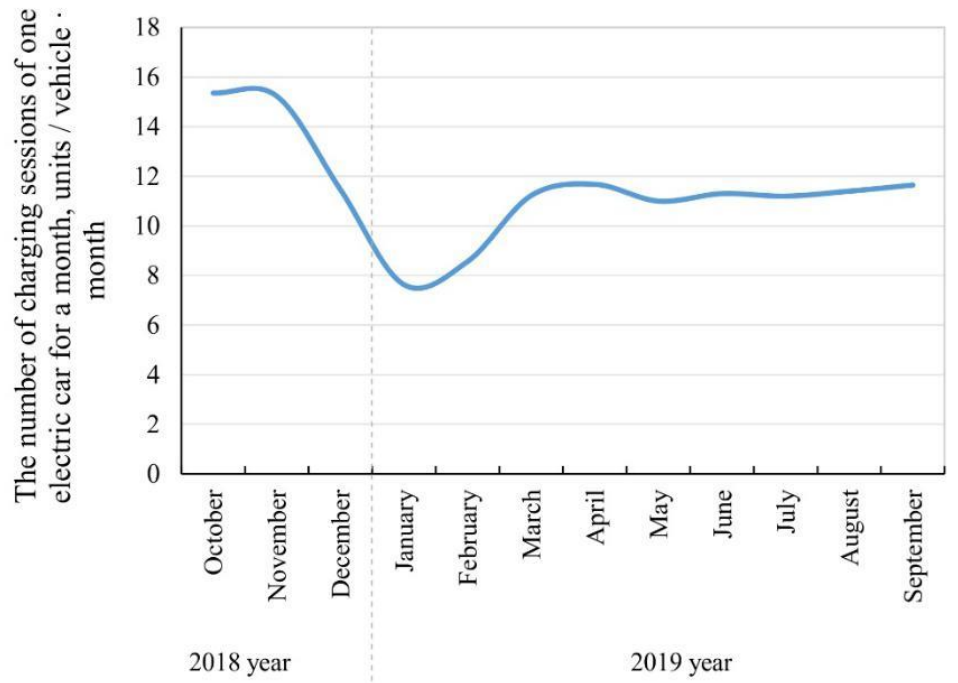

Fig. 4. The dynamics of changes in the number of charging sessions of one electric car during the month in Tyumen.

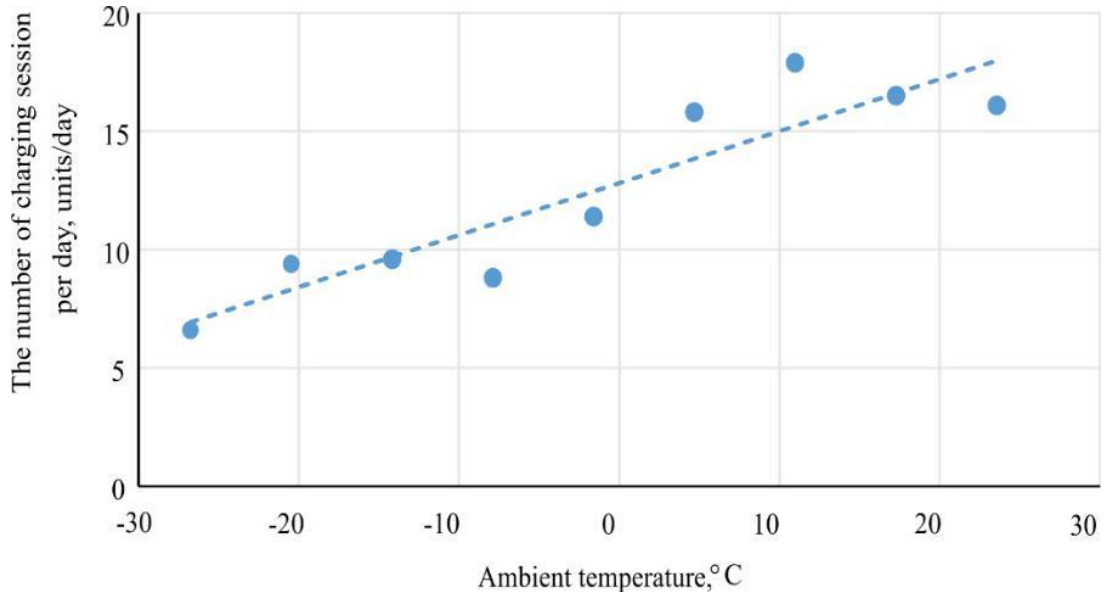

Fig. 5. The pattern of changes in the number of charging sessions per day from the ambient temperature. 
As it was previously established, a decrease in the ambient temperature leads to a decrease in the number of charging sessions by $59 \%$, which is due to an increase in the duration of the charging session and a decrease in the amount of energy transferred during one operation, which is shown in figure 6 and figure 7.

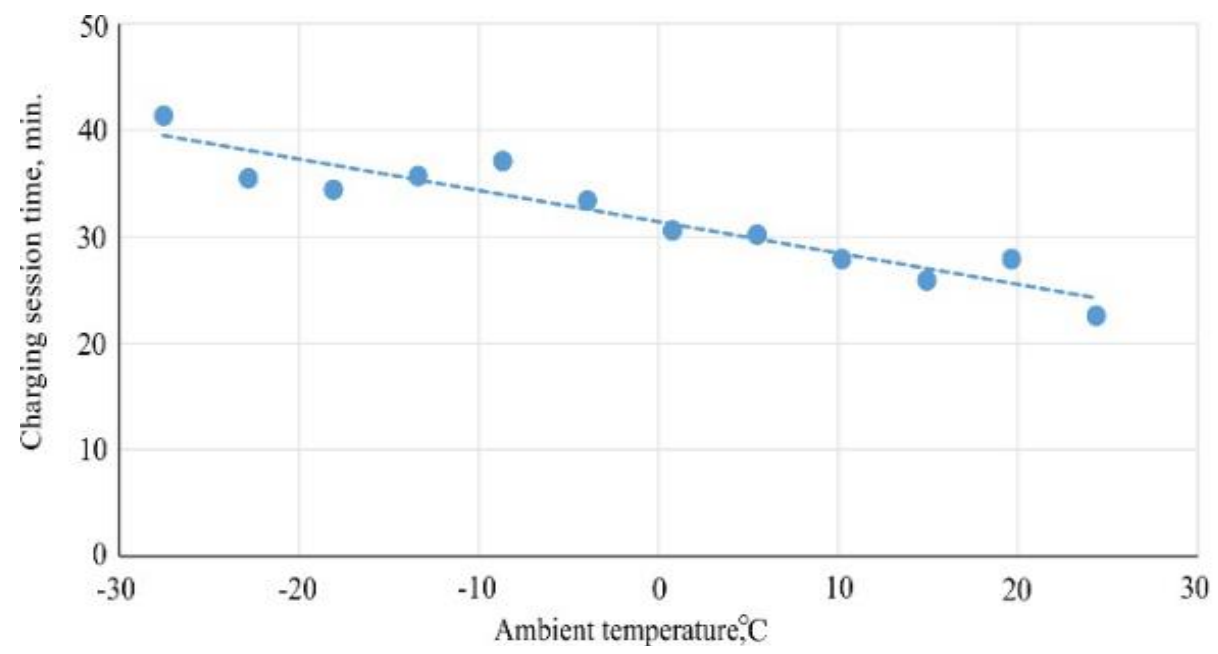

Fig. 6. The pattern of change in the time of the charging session from the ambient temperature.

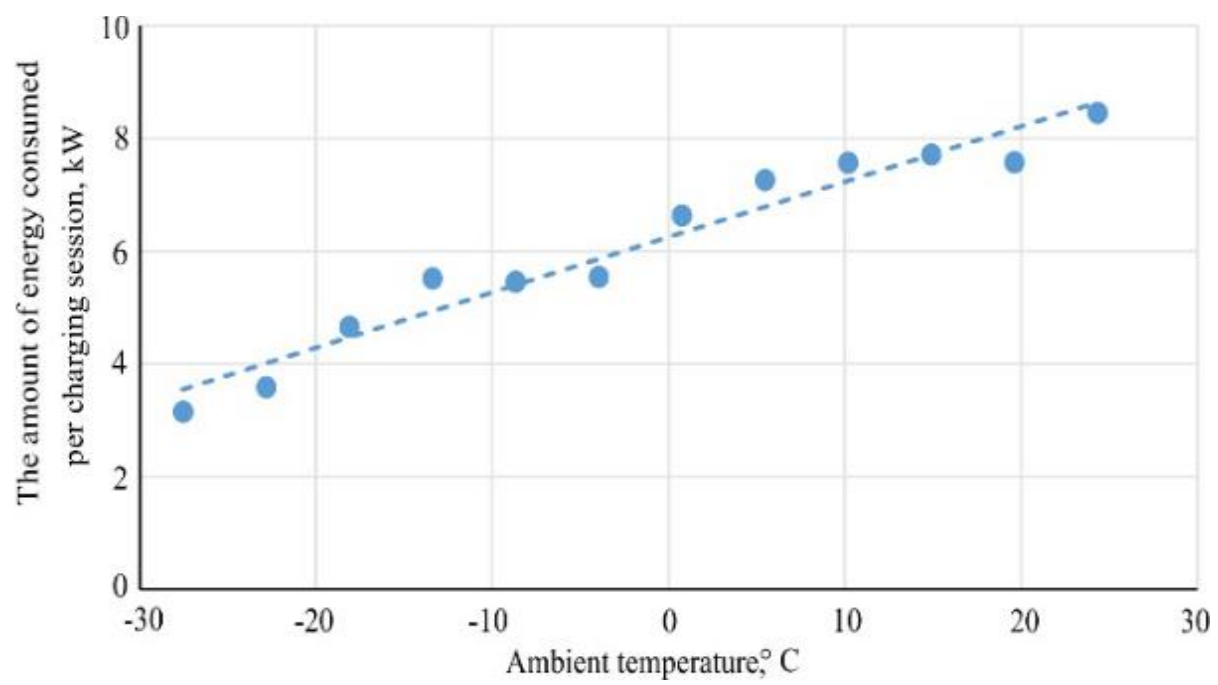

Fig. 7. The pattern of changes in the amount of energy transferred during one charging session from the ambient temperature.

As a result of the analysis of the close connections between the time of the charging session and air temperature, as well as the amount of energy transferred per charging session, the pair correlation coefficients are calculated, which are respectively 0.94 and 0.97 at a significance level of 0.95 . A decrease in the amount of energy transferred with an increase in the duration of the charging session leads to a decrease in the charging rate with a decrease in ambient temperature, which is shown in figure 8. 


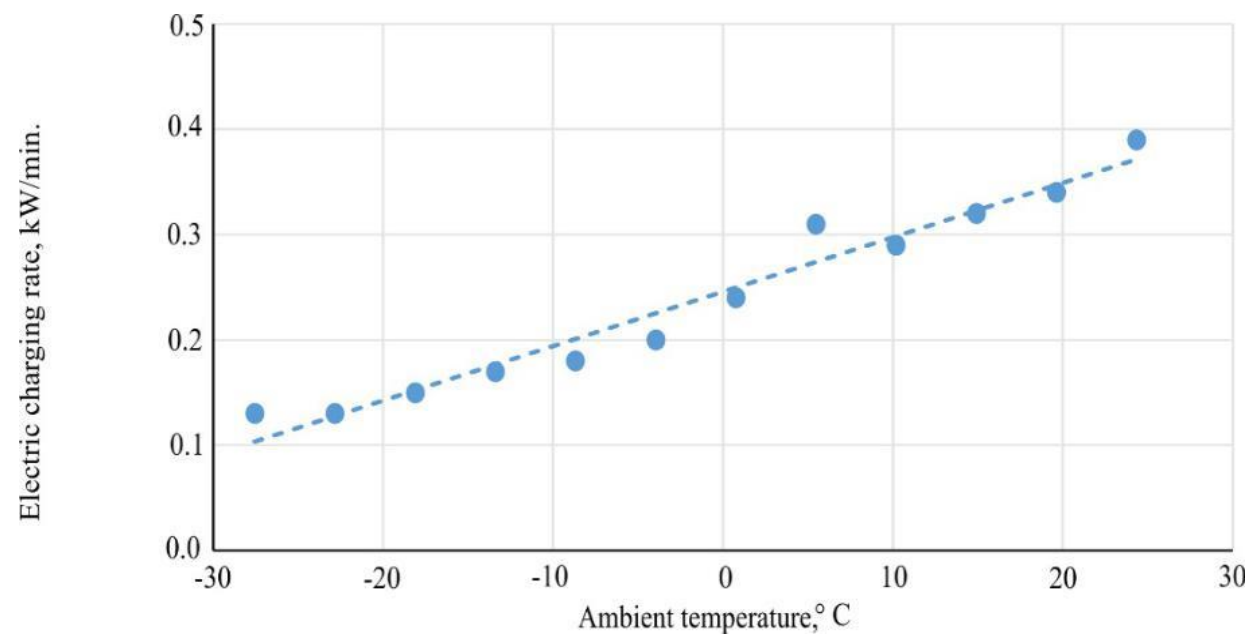

Fig. 8. The pattern of changes in the charging speed of an electric vehicle from the ambient temperature.

A decrease in ambient temperature leads to a decrease in the charging speed of electric vehicles in the winter by $62 \%$, which is the reason for the decrease in the number of charging sessions under low temperature conditions. The close connection between the charging speed of the electric vehicle and the ambient temperature was estimated using the pair correlation coefficient, which was 0.98 with a significance level of 0.95 .

\section{Conclusion}

The development of electric vehicles, which is now observed in the Russian Federation, leads to the need to create a charging infrastructure. Variable operating conditions, and in particular low-temperature, cause difficulties in the use of electric vehicles, which are associated with a low power reserve and increased energy consumption in the winter. These features of the operation of electric vehicles in the Russian Federation can lead to an increase in the number of charging stations needed to create a developed infrastructure, and, therefore, can increase capital costs.

During the processing of the initial data, patterns were revealed in the change in the number of charging sessions from the ambient temperature, a decrease in which causes a decrease in the number of charging sessions by $59 \%$. To identify the causes of this phenomenon, an analysis of the charging station parameters at various ambient temperatures was made. It showed that lowering the ambient temperature leads to an increase in the charging time of an electric vehicle by $46 \%$ while reducing the amount of energy transmitted by $60 \%$. As a result of calculating the charging speed of the electric vehicle, it was revealed that a decrease in air temperature causes a decrease in speed by 4 times.

The obtained patterns and the results of studying the initial data will allow us to develop a methodology for calculating the number of charging stations required in variable climatic conditions of operation of electric vehicles.

\section{Reference}

1. S. Brown, D. Pyke, P. Steenhof, Energy Policy 38, 3797 - 3806 (2010)

2. M. Coffman, P. Bernstein, S. Wee, Transport Reviews 1080, 1217282 (2016) 
3. M. Kuby, S. Lim, Networks and Spatial Economics 7, 129-152 (2006)

4. Y.-W. Wang, C.-R. Wang, Transport. Res. Part E: Log. and Transport. Rev. 46, 791801 (2010)

5. M. Frick, K. Axhausen, G. Carle, A. Wokaun, Transport. Res. Part D 12, 10-22 (2007)

6. I. Frade, A. Ribeiro, A. Goncelves, A. Antunes, Trasport. Res. Record J. of the Trans. Res. Board 2252, 91-98 (2011)

7. S. He, Y. Kuo, D. Wu, China Transport. Res. Part C 67, 131-148 (2016)

8. M. Ghamami, Y. Nie, A. Zockaie, Inter. J. of Sustain. Transport. 10, 343-353 (2015)

9. D. Gimenez - Gaydou, A. Ribeiro, J. Gutierrez, A. Antunes, Inter. J. of Sustain. Transport. 10, 393-405 (2014)

10. J. Ko, J. Shim, Inter. J. of Sustain. Transport. 10, 139-146 (2014)

11. M. Pternea, K. Kepaptsoglou, M. Karlaftis, Transport. Res. Part A 77, 276-291 (2015)

12. N. Sathaye, S. Kelley, Transport. Res. Part E: Log. and Transp. Rev. 59, 15-33 (2013)

13. F. He, D. Wu, Y. Yin, Y. Guan, Transport. Res. Part B: Meth. 47, 87-101 (2013)

14. O. Berman, Z. Drezner, D. Krass, Comp. and Operat. Res. 37, 1675-87 (2010)

15. H.-Y. Mak, Y. Rong, Z.-.J M. Shen, Management Science 59, 1557-75 (2013)

16. D.A. Gura, I.G. Markovskii, B.A. Hahuk, S.K. Pshidatok, IOP Conference Series: Materials Science and Engineering 698(4), 044014 (2019). DOI: 10.1088/1757899X/698/4/044014

17. V. Shishkina, D. Gura, I. Gribkova, M. Bykova, IOP Conference Series: Materials Science and Engineering 698(6), 066016 (2019). DOI: 10.1088/1757899X/698/6/066016

18. Manakov, A., Kolarzh, S., Mashkov, A., MATEC Web of Conferences, 239, 272018 , 04002, DOI: $10.1051 /$ matecconf $/ 201823904002$ 